\title{
Anabases
}

ANABASES Traditions et réceptions de l'Antiquité

17 | 2013

Varia

\section{Friedrich August Wolf, « héros éponyme » de la Science de l'Antiquité}

\section{Anthony Andurand}

\section{(2) OpenEdition}

1 Journals

Édition électronique

URL : http://journals.openedition.org/anabases/4209

DOI : 10.4000/anabases.4209

ISSN : 2256-9421

\section{Éditeur}

E.R.A.S.M.E.

\section{Édition imprimée}

Date de publication : 1 mars 2013

Pagination : 223-229

ISSN : 1774-4296

\section{Référence électronique}

Anthony Andurand, «Friedrich August Wolf, « héros éponyme » de la Science de l'Antiquité », Anabases [En ligne], 17 | 2013, mis en ligne le 01 avril 2016, consulté le 20 octobre 2019. URL : http:// journals.openedition.org/anabases/4209; DOI : 10.4000/anabases.4209 
Anabases 17 (2013), p. 223-229.

\section{Friedrich August Wolf, " héros éponyme " de la Science de l'Antiquité}

ANTHONY ANDURAnd

Pourquor relire la Darstellung der Alterthums-Wissenschaft de l'helléniste allemand Friedrich August Wolf (1759-1824) ? Dans quelle mesure peut-on considérer cette œuvre, plus de deux siècles après sa parution, comme un classique de la science de l'Antiquité ? L'ouvrage, l'histoire de sa genèse et de sa réception dans le champ des études anciennes sont d'abord étroitement liés à une époque, sur laquelle les documents présentés ici - la dédicace à Goethe et la préface de l'édition originale, datée du mois de juillet 1807 - peuvent apporter un éclairage précieux.

Le destin de la Darstellung s'élabore en effet à la croisée d'une pluralité de contextes, qui conferent à la démarche wolfienne sa tonalité et sa signification particulières ${ }^{1}$.

1 La Darstellung a fait l'objet, ces dernières décennies, d'une ample littérature scientifique. S. Cerasuolo a proposé il y a quelques années, en guise d'introduction à l'édition italienne de l'ouvrage, une remarquable synthèse : "Wolf teorico della filologia classica ", in F.A. Wolf, Esposizione della scienza dell'antichità, Naples, Bibliopolis, 1999, p. 13-97. On se reportera également à l'ouvrage collectif paru la même année : S. CerASUOLO (a cura di), Friedrich August Wolf e la scienza dell'antichità. Atti del convegno internazionale (Napoli 24-26 maggio 1995), Naples, Pubblicazioni del Dipartimento di Filologia Classica dell'Università degli Studi, 1999. Sur la place de la Darstellung dans la démarche et l'œuvre du philologue : M. FunRmann, "Friedrich August Wolf zur 200. Wiederkehr seines Geburtstages am 15. Februar 1959 ", Deutsche Vierteljahrsschrift für Literaturwissenschaft und Geistesgeschichte, 33. Jahrgang, 2 (1959), p. 187-236 ; A. Grafton, " Prolegomena to Friedrich August Wolf ", Journal of the Warburg and Courtauld Institutes 44 (1981), p. 101-129 ; S. Marchand, Down from Olympus. Archaeology and Philhellenism in 
Celui des guerres napoléoniennes, tout d'abord, dont les pages qui précèdent l'ouvrage portent la trace visible. Le projet de la Darstellung est conçu au lendemain des défaites d'Iéna et d'Auerstaedt (14 octobre 1806). Vécus, dans la mémoire prussienne et allemande, comme un véritable traumatisme, ces événements marquent aussi une profonde césure dans la carrière de Wolf : dès le 17 octobre, Halle est occupée par les troupes napoléoniennes ; le général Bernadotte, trois jours plus tard, fait fermer l'université de la ville, où le philologue était titulaire, depuis 1783, d'une chaire de philosophie et de pédagogie. Libéré de ses obligations universitaires, Wolf met à profit cette période d'inactivité contrainte pour composer une "introduction " (Einleitung) aux leçons sur l'Encyklopädie und Methodologie der Studien des Alterthums tenues à l'université de Halle depuis 1785. La matière de cet "essai » (Aufsatz) est élaborée durant l'hiver 1806-1807. L'ouvrage est publié en 1807 à Berlin, où Wolf s'est installé à l'automne, sous le titre complet de "Présentation de la science de l'Antiquité d'après son concept, son étendue, son but et sa valeur " (Darstellung der Alterthums-Wissenschaft nach Begriff, Umfang, Zweck und Werth). Il constitue le premier volume de la revue Museum der Alterthums-Wissenschaft, spécialement créée pour l'occasion par Wolf et le philologue Philipp Buttmann ${ }^{2}$.

Que Wolf ait choisi, selon les mots empruntés à la préface, de se tenir à l'écart des "tourments du temps " pour composer son ouvrage ne signifie pas, loin s'en faut, que la composition de celui-ci n'est pas directement liée aux événements de l'année 1806. "L'État, aurait déclaré Frédéric-Guillaume III à Königsberg en 1807, doit remplacer par des forces spirituelles les forces physiques qu'il a perdues ${ }^{3}$. " Une ambition similaire anime, la même année, le projet wolfien : au lendemain de l'effondrement de la Prusse, humiliée par les troupes napoléoniennes et amputée, par la paix de Tilsit ( 9 juillet 1807), de près de la moitié de ses territoires, la Darstellung participe, dans le

Germany, 1750-1970, Princeton, Princeton University Press, 1996, p. 16-24 ; S. Maufroy, "Friedrich August Wolf, un modèle philologique et ses incidences européennes ", Revue Germanique Internationale 14 (2011), p. 27-39 (en particulier p. 36-39). Nous avons consulté avec grand profit, enfin, les pages que G. Leghissa, dans ses travaux sur la place de la modernité dans l'imaginaire de la science allemande du XIX e siècle, consacre à Wolf : Incorporare l'antico. Filologia classica e invenzione della modernità, Milano, Mimesis Edizioni, 2007, p. 25-47 (sur la valeur fondatrice de l'œuvre wolfienne) et p. 49-55 (sur la dédicace à Goethe), ainsi que "L'Antiquité grecque comme miroir de la Deutschtum ", in D. Foucault, P. Payen (dir.), Les Autorités. Dynamiques et mutations d'une figure de référence à l'Antiquité, Grenoble, Jérôme Millon, 2007, p. 213-221.

2 La revue fondée par Wolf et Buttmann ne fit paraître que deux numéros, parus l'un en 1807, l'autre, en deux livraisons, en 1808 et 1810 . Le second volume accueille notamment l'étude d'A. Bоескн, "Über die Versmaße des Pindaros ", in Museum der Alterthums-Wissenschaft, Zweiter Band, 1808, p. 168-362.

3 "Der Staat muß durch geistige Kräfte ersetzen, was er an physischen verloren hat ». La formule est reproduite chez A. GARZYA, "Introduzione ", in A. BOECKH, La filologia come scienza storica, Napoli, Guida Editori, 1987, p. 7. 
champ de la science et de la culture, de l'effort de redressement du Royaume et de la nation allemande. Dans ce contexte, l'œuvre se propose, ainsi que le suggère la dédicace à Goethe, de transmettre, comme un " héritage impérissable ", le "palladium " que représentent les savoirs sur l'Antiquité, dans l'espoir d'un relèvement spirituel de la " patrie " meurtrie et menacée " par des mains impies ». La Darstellung de Wolf, dans cette perspective, peut apparaître comme le prodrome des réformes scolaires et universitaires initiées, entre 1809 et 1810, par Wilhelm von Humboldt, alors en charge de la Sektion für Kultus und Unterricht du Ministère prussien de l'Intérieur.

Contribution à l'entreprise de reconstruction et de consolidation de la jeune nation allemande, la Darstellung se veut aussi - c'est le deuxième contexte que nous devons envisager - une ouvre de refondation dans le champ des savoirs sur l'Antiquité. Au moment où paraît son essai, Wolf est déjà au faîte de sa gloire. Ses fameux Prolegomena ad Homerum, parus à Halle en 1795, lui valurent une solide réputation dans le domaine de la critique textuelle et historique. En 1807, le savant est considéré, à l'échelle de l'érudition européenne, comme la figure de proue de la philologie allemande. Les spécialistes de la Wissenschaftsgeschichte, à vrai dire, ont parfois vu dans la Darstellung - comme du reste dans la légende qui entoure l'inscription de Wolf comme studiosus philologiae, en 1777, à l'université de Göttingen ${ }^{4}$ - l'acte de naissance de la science de l'Antiquité (Altertumswissenschaft). Le savant fut longtemps présenté comme celui qui, dans un geste inaugural, dota ex nibilo la discipline tout à la fois d'un nom et d'un projet intellectuel. Les travaux menés depuis plusieurs décennies dans ce dossier historiographique ont permis non de relativiser l'importance de la Darstellung dans l'histoire des études anciennes, mais de mettre en lumière l'apport, dans le projet wolfien, des traditions savantes et intellectuelles héritées du siècle précédent - telles qu'elles se façonnent notamment, dans la seconde moitié du XVIII ${ }^{\mathrm{e}}$ siècle, au Seminarium philologicum de l'université de Göttingen, sous l'impulsion de Johann Matthias Gesner ou Christian Gottlob Heyne ${ }^{5}$. Si Wolf, par conséquent, ne saurait être considéré comme le génial inventeur de l'Altertumswissenschaft, son principal mérite reste d'avoir su réunir les traditions et les orientations nouvelles de la discipline en un projet cohé-

4 Dès 1913, E. Schröder montrait, à partir d'un travail d'archives, que l'épisode relevait bien de la légende : Wolf fut loin d'être le premier à s'inscrire à Göttingen comme studiosus philologiae. Dès 1736, avant même la création du Seminarium philologicum, un certain Johann Heinrich Wagner s'inscrivait sous ce titre. Voir E. SCHRÖDER, «Studiosus philologiae ", Neue Jahrbücher für das klassische Altertum 32 (1913), p. 168-171.

5 Ces recherches ont permis de faire apparaittre ce que la réflexion de Wolf sur le projet théorique de la science de l'Antiquité et la constitution de celle-ci en une discipline autonome doivent à ses prédécesseurs - une dette longtemps occultée par Wolf lui-même et par l'historiographie du XIX ${ }^{\mathrm{e}}$ siècle. Sur cet aspect du problème, voir en particulier le bilan que dresse A. Grafton, "Prolegomena to Friedrich August Wolf », p. 109, et les remarques de S. Fornaro, I Greci senza lumi. L'antropologia della Grecia antica in Christian Gottlob Heyne (1729-1812) e nel suo tempo, Göttingen, Vandenhoeck und Ruprecht, 2004, p. 111 et p. 122-123. 
rent et systématique, dont il s'applique, dans les pages de la Darstellung, à préciser les contours et les ambitions. C'est là le but, conformément au programme esquissé dans la préface de l'édition originale, que Wolf assigne à son ouvrage : proposer une "vision d'ensemble " (Übersicht) de la science de l'Antiquité, dans son " tout organique " (organisches Ganz) comme dans chacune des "parties" (Teile) qui la composent, pour élever la discipline à la dignité d'une "science bien ordonnée " (wohlgeordnete Wissenschaft). La définition que Wolf, dans les premières pages de son essai, formule de l'Altertumswissenschaft, maintes fois reprise par les hellénistes allemands du XIX siècle, est passée à la postérité :

"Si après cela on exige encore de nous une description plus précise du tout de notre science, nous dirons qu'elle concerne l'ensemble des savoirs et des informations qui nous font connaître les actions et les destinées, l'état politique, intellectuel et domestique des Grecs et des Romains, leur culture, leur langue, leurs arts et leurs sciences, leurs coutumes, leur religion, leur caractère national et leur façon de penser, de sorte que nous soyons à même de comprendre à fond les œuvres qui nous sont parvenues d'eux et de les apprécier en prêtant attention à leur contenu comme à leur esprit, en réactualisant la vie antique et en la comparant à celle des époques ultérieures ou à celle de l'époque d'aujourd'hui ${ }^{6}$. "

L'auteur de la Darstellung délimite ainsi les contours de l'objet de la discipline dont il se fait le promoteur, comprise comme l'étude de la vie des Grecs et des Romains ${ }^{7}$, dans toutes ses dimensions, même les plus prosaïques et les plus concrètes. Il détermine, dans le même temps, les principes d'une méthode, ancrée dans l'étude raisonnée, critique et systématique de toutes les sources anciennes et la collaboration, à l'intérieur d'un projet commun, de l'ensemble des disciplines qui ont trait à l'Antiquité (philologie, archéologie, épigraphie, numismatique, "mythologie ", etc). Cet effort de réactualisation, de "présentification " (Vergegenwärtigung) de la vie antique est adossé, en dernière instance, à un dessein plus élevé encore, formulé à rebours de toute préoccupation utilitariste : celui de participer à la Bildung de la jeunesse et de la nation allemandes, d'œuvrer à cette "formation harmonieuse de l'esprit et du cœur »

6 F.A. Wolf, Darstellung der Alterthums-Wissenschaft, Berlin, Realschulbuchhandlung, 1807, p. 30 : "Wird hienach noch eine nähere Beschreibung des Ganzen unserer Wissenschaft gefordert, so wird sie auf den Inbegriff der Kenntnisse und Nachrichten gehen, die uns mit den Handlungen und Schicksalen, mit dem politischen, gelehrten und häuslichen Zustande der Griechen und Römer, mit ihrer Cultur, ihren Sprachen, Künsten und Wissenschaften, Sitten, Religionen, National-Charakteren und Denkarten bekannt machen, dergestalt daß wir geschickt werden, die von ihnen auf uns gekommenen Werke gründlich zu verstehen und mit Einsicht in ihren Inhalt und Geist, mit Vergegenwärtigung des alterthümlichen Lebens und Vergleichung des spätern und des heutigen, zu genießen».

7 Des Grecs et des Romains, et d'eux seuls : l'Altertumswissenschaft, dès les premiers temps et jusque dans les premières décennies du $\mathrm{Xx}^{\mathrm{e}}$ siècle, s'est toujours comprise comme klassische Altertumswissenschaft, science de l'Antiquité « classique ». 
(harmonische Ausbildung des Geistes und Gemüths) que le philologue évoque dans les dernières lignes de la préface. On retrouve là l'essentiel du programme néo-humaniste et les principes forgés à la même époque par Wilhelm von Humboldt, que l'on peut tenir pour le théoricien de la notion de Bildung. De fait, les deux savants, formés à la philologie classique au Seminarium philologicum de Heyne, ont entretenu à partir des années 1790 une amitié durable et une étroite collaboration scientifique. S'agissant des visées des études anciennes et, plus particulièrement, des études grecques, le projet élaboré dans la Darstellung est conforme aux vues développées dans ce que J. Quillien appelle l' " anthropologie philosophique ${ }^{8}$ " humboldtéenne : modèle d'une humanité (Menschheit) accomplie, la Grèce est conçue comme l'instrument privilégié de la connaissance et de la formation de l'homme en général ${ }^{9}$.

Ainsi, la Darstellung, à l'image du dialogue entre Wolf et Humboldt dont elle est l'occasion, constitue un témoignage de première importance pour comprendre - c'est le troisième et dernier contexte que nous souhaitons évoquer - les liens qui unissent alors, sous la bannière de l'hellénisme, les milieux savants et les représentants du classicisme allemand. La dédicace à Goethe, de ce point de vue, élevé au rang de " témoin » et " protecteur " du programme tracé dans l'ouvrage de 1807, dit bien la nature des relations, des idéaux et des codes qui régissent à cette époque l'érudition et les lettres allemandes. Les pages placées au seuil de la Darstellung manifestent la profonde admiration du savant et l'amitié sincère qui l'unit au poète, une amitié née plus de dix ans plus tôt, lors de la publication des Prolegomena ad Homerum. Le sage de Weimar n'est d'ailleurs pas étranger au projet de la Darstellung. Dans une lettre datée du 28 novembre 1806, quelques semaines après le désastre d’Iéna, Goethe avait invité son ami à mettre par écrit et à publier, sous la forme d'une " ébauche condensée " (compendiarischer Entwurf), ses leçons halloises ${ }^{10}$. Quelques mois plus tard, Wolf prend soin de rappeler, dans la dédicace de l'ouvrage, l'impulsion décisive qu'a représentée, au moment de concevoir l'entreprise de la Darstellung, cette " sollicitation amicale " (freundlischer Zuruf).

L'hommage solennel rendu dans la Widmung est à la mesure de la vénération qu'éprouve Wolf pour le héros de la Goethezeit. Goethe s'y voit décerner à deux reprises le titre de "connaisseur " et " représentant " de l'esprit grec, de cet esprit qui prit chez le poète comme une "seconde demeure ". Le compliment, en vérité, n’a rien d'original et apparaît même comme un topos des lettres allemandes de la seconde

8 J. Quillien, L'anthropologie philosophique de G. de Humboldt, Lille, Presses Universitaires de Lille, 1991.

9 Sur les relations entre Wolf et Humboldt et la place de l'Antiquité grecque dans le projet de Bildung : J. Quillien, G. de Humboldt et la Grèce. Modèle et histoire, Lille, Presses Universitaires de Lille, 1991, p. 15-22.

10 Goethes Briefe an Friedrich August Wolf, M. Bernays (Hg.), Berlin, Verlag von G. Reimer, 1868 , p. 111. 
moitié du XVIII ${ }^{e}$ siècle ${ }^{11}$. Herder et Goethe avaient déjà salué en Winckelmann un Grec parmi les Modernes ${ }^{12}$; c'est ainsi, également, que Schiller avait célébré Goethe, et Humboldt, à son tour, Schiller lui-même ${ }^{13}$. Plus audacieuse, en revanche, s'avère l'utilisation que Wolf, toujours dans la dédicace, propose du thème de la parenté entre Grecs et Allemands, emprunté une fois encore à Humboldt ${ }^{14}$. Le motif de l' "affinité " (Verwandtschaft) spirituelle avec les Grecs acquiert en effet dans ce texte, comme l'a remarqué $\mathrm{G}$. Leghissa ${ }^{15}$, une valeur " métonymique » : il vaut pour le poète comme pour la nation dont il est le digne représentant et la figure tutélaire, la nation allemande. De même que Goethe est célébré comme une manière de réincarnation du Geist hellénique, de même les Allemands sont décrits comme des Grecs de l'époque moderne, et l'Allemagne, en vertu de cette affinité proclamée, semble appelée à devenir une nouvelle Hellade. Ce discours, développé presque incidemment dans la dédicace, imprègne d'une tonalité singulière le programme scientifique proposé dans la Darstellung. Dignes héritiers des anciens Hellènes, les Allemands, écrit Wolf, sont les seuls, parmi les Modernes, à pouvoir, après les "déformations " (Verbildungen) dont l'histoire et la culture helléniques n'ont cessé d'être l'objet, s'accorder parfaitement " aux modes du chant et de la déclamation des Grecs »; capables d'entrer en résonance avec l'esprit grec, poursuit-il, les Allemands peuvent aspirer à devenir, conformément à la parenté qui les relie à ces ancêtres spirituels, "les plus profonds chercheurs et exégètes » de la beauté et de la vérité contenues dans l'héritage hellénique. Légitimant ainsi la supériorité de l'Altertumswissenschaft et la position rectrice qu'elle ambitionne d'occuper dans l'érudition européenne, le motif de l'affinité avec les Grecs institue également, dans l'économie de la Darstellung, la vocation des hellénistes allemands et

11 Sur l'histoire et la signification de ce topos littéraire : M. FuHRMAnN, «Die "Querelle des Anciens et des Modernes", der Nationalismus und die deutsche Klassik », in R.R. BolgaR (ed.), Classical Influences on Western Thought, 1650-1870, Cambridge, Cambridge University Press, 1978, p. 107-129 (voir en particulier p. 122-129).

12 J.G. vON Herder, Denkmal Johann Winckelmanns [1778], in Sämmtliche Werke, B. Suphan (Hg.), Berlin, Weidmann, VIII, p. 482 ; J.W. von Goethe, Skizze zu einer Schilderung Winckelmanns [1805], trad. J.E. Jackson, Esquisse d'un portrait de Winckelmann, Neuchâtel, Éd. de la Baconnière, 1980, p. 46 et 47.

13 F. SCHILler, lettre à Goethe (23 août 1794), in Goethe-Schiller. Correspondance 17841805, C. RoËLS (éd.), Paris, Gallimard, 2 vol., 1994, I, p. 45 ; W. von Humboldt, lettre à Schiller (6 novembre 1795), in Briefe, München, Carl Hanser Verlag, 1952, p. 117.

14 Sur la genèse de ce motif chez Humboldt et son histoire au XIX ${ }^{\mathrm{e}}$ siècle : M. LANDFESTER, "Griechen und Deutsche : der Mythos einer "Wahlverwandtschaft" ", in H. Berding (Hg.), Studien zur Entwicklung des kollektiven Bewußtseins in der Neuzeit, Frankfurt am Main, Suhrkamp, 1996, 3 vol., III, p. 198-219 ; F. SAURE, "Meine Grille von der Ähnlichkeit der Griechen und der Deutschen". Nationalkulturelle Implikationen in Wilhelm von Humboldts Antikekonzept ", in V. Ronsenberger (Hg.), Die Ideale der Alten. Antikerezeption um 1800, Stuttgart, F. Steiner Verlag, 2008, p. 113-129. 
de l'entreprise dont Wolf se veut l'initiateur : celle de "féconder " (befruchten), par la transmission des savoirs sur le passé grec, l'" esprit " (Geist) de la nation allemande et de la guider vers l'accomplissement de son destin de nouvelle Hellade.

Miroir des infortunes et des aspirations de l'Allemagne du début du XIXe siècle, manifeste des ambitions de la nouvelle science de l'Antiquité, la Darstellung acquit très tôt, outre-Rhin, une place toute singulière dans les annales de l'érudition ${ }^{16}$. Jusque dans les premières décennies $\mathrm{du} \mathrm{Xx}^{\mathrm{e}}$ siècle, en effet, l'auteur de ce véritable monument des études grecques fut honoré tantôt comme " le fondateur de la philologie comme science particulière et autonome ${ }^{17}$ " (Paulsen), tantôt comme le " héros éponyme de la race des philologues allemands ${ }^{18}$ " (Niebuhr). À l'heure où la Goethezeit tendait à s'identifier, dans le domaine de la littérature, avec l'idée d'une deutsche Klassik, la Darstellung, elle, perpétuait le souvenir de cette Wolfzeit conçue, dans le regard rétrospectif des antiquisants, comme "l'âge d'or de la philologie ", comme "la période classique de la science de l'Antiquité ${ }^{19}$ ». C'est à ce titre que l'on peut relire la Darstellung et les textes présentés ici : moins comme le point de départ d'une tradition savante à laquelle l'historien de l'Antiquité serait encore directement relié, que comme le témoignage vivant d'une époque devenue, dans la mémoire de la science allemande, héroïque et fondatrice.

\begin{tabular}{l} 
Anthony ANDURAND \\
\hline PLH-ERASME \\
Pavillon de la recherche \\
5, allées Antonio Machado \\
F-31058 Toulouse cedex 9 \\
anthonyandurand@yahoo.fr
\end{tabular}

16 P. Hummel a rassemblé sur cette question un précieux dossier documentaire, qu'elle analyse avec finesse dans son Histoire de l'histoire de la philologie, Genève, Droz, 2000, p. 106-115. Voir également les pages suggestives de S. Maurroy, «Friedrich August Wolf, un modèle philologique ", p. 27-31.

17 F. Paulsen, Geschichte des gelehrten Unterrichts, Leipzig, Verlag von Veit \& Comp., 1885, p. 530.

18 B.G. Niebuhr, «Die Sikeler in der Odyssee » [1827], in Kleine historische und philologische Schriften, Bonn, E. Weber, 2 vol., 1828-1843, II, p. 227.

19 W. Herbst, Das classische Alterthum in der Gegenwart, Leipzig, Teubner, 1852, p. 13. Nous soulignons. 